\title{
Intervalos de referencia en parámetros sanguíneos indicadores del balance mineral para grupos de vacas lecheras en el sur de Chile
}

\author{
Reference intervals for blood markers of mineral balance for groups \\ of dairy cows in the South of Chile \\ C Wagemann ${ }^{\mathrm{a}}$, F Wittwer ${ }^{\mathrm{b}}, \mathrm{R}_{\text {Chihuailaf }}$, M Noro ${ }^{\mathrm{b}, \mathbf{d}^{*}}$ \\ ${ }^{a}$ Escuela de Graduados, Facultad de Ciencias Veterinarias, Universidad Austral de Chile, Valdivia, Chile. \\ 'Instituto de Ciencias Clínicas Veterinarias, Facultad de Ciencias Veterinarias, Universidad Austral de Chile, Valdivia, Chile. \\ ${ }^{\mathrm{E} E s c u e l a ~ d e ~ M e d i c i n a ~ V e t e r i n a r i a, ~ U n i v e r s i d a d ~ C a t o ́ l i c a ~ d e ~ T e m u c o, ~ T e m u c o, ~ C h i l e . ~}$ \\ dUniversidade Federal do Pampa, Uruguaiana-RS, Brasil.
}

\begin{abstract}
SUMMARY
The aim of this study was to propose reference intervals (RI) for blood markers of mineral balance in prepartum and lactating groups of dairy cows in the South of Chile. The blood concentrations values of calcium, inorganic phosphate, magnesium, sodium, potassium, copper and zinc and ratio calcium/inorganic phosphate were analysed. The values were obtained from 1,414 metabolic profiles performed between 2003 and 2011 to groups of cows from dairy herds. Each metabolic profile consisted of a group of 5 or more cows, and according to their physiological condition they were classified in 2 categories: prepartum $(n=380)$ or lactating $(n=1,034)$. The means of the analytes were calculated for each group and category. The distribution of the data was analyzed and non-parametric values were log-transformed. For each analyte outliers were identified and removed. RI were estimated with a non-parametric test, based on the $2.5^{\text {th }}$ and $97.5^{\text {th }}$ percentiles when the sample size was $\geq 120$, and with a robust method when the sample size was $40 \leq n$ $\leq 120$. The RI for most of the blood markers were similar to those reported previously by the laboratory, except for inorganic phosphate established for both categories and of copper for the prepartum category. The results of this study will improve the interpretation of metabolic profiles and its accuracy to assess the metabolic and nutritional status of prepartum and lactating groups of cows of dairy herds in the South of Chile.
\end{abstract}

Key words: reference intervals, mineral balance, dairy cows, metabolic profiles.

\section{RESUMEN}

El objetivo del estudio fue proponer intervalos de referencia (IR) de parámetros sanguíneos indicadores del balance mineral para grupos de vacas preparto y en lactación de rebaños lecheros del sur de Chile. Se utilizaron los valores de las concentraciones sanguíneas de calcio, fosfato inorgánico, magnesio, sodio, potasio, cobre y zinc y el cociente calcio:fosfato inorgánico, obtenidos de 1.414 perfiles metabólicos realizados a grupos de vacas de rebaños lecheros entre los años 2003 y 2011. Cada perfil estuvo conformado por un grupo de 5 o más vacas y de acuerdo a su estado fisiológico se clasificaron en 2 categorías: preparto $(\mathrm{n}=380)$ y lactación $(\mathrm{n}=1.034)$. Las medias de los parámetros fueron calculadas para cada grupo y categoría. Se analizó la distribución de los datos, siendo los no paramétricos normalizados por el método de logaritmos. Para cada parámetro se identificaron y eliminaron los outliers. Los IR se determinaron con una prueba no paramétrica, en base a los percentiles 2,5 y 97,5, cuando el tamaño muestral fue $\geq 120$ y con un método robusto cuando el tamaño muestral fue $40 \leq n \leq 120$. Los IR de la mayoría de los parámetros fueron similares a los reportados previamente por el laboratorio, a excepción de los IR de fosfato inorgánico para las categorías preparto y lactación y de cobre para la categoría preparto. Los resultados pretenden contribuir a mejorar la exactitud de los perfiles metabólicos para evaluar el estado metabólico nutricional de grupos de vacas de rebaños lecheros en el sur de Chile.

Palabras clave: intervalos de referencia, balance mineral, vacas lecheras, perfiles metabólicos.

\section{INTRODUCCIÓN}

Los minerales son nutrientes esenciales para los bovinos y sus concentraciones en el organismo deben fluctuar dentro de intervalos estrechos con el objeto de mantener el adecuado estado sanitario y productivo de los rebaños (NRC 2001, Wittwer 2007). Los desbalances minerales se presentan con frecuencia en las vacas lecheras, limi-

Aceptado: 02.05.2013.

* Casilla 567 Valdivia, Chile. mirelanoro@gmail.com tando su productividad y constituyendo un tema de preocupación asociado al bienestar animal. Sin embargo, sus signos generalmente no son específicos y pueden confundirse con otras alteraciones que comúnmente afectan a los rebaños (Khan y col 2004, Wittwer 2007). Por este motivo, para evaluar el estado metabólico nutricional de los individuos o rebaños, se determinan las concentraciones sanguíneas de minerales mediante el empleo de perfiles metabólicos (Herdt y col 2000, Van Saun 2010).

La identificación de los desbalances minerales en los rebaños lecheros se basa en comparar los valores me- 
dios de las concentraciones de algunos parámetros de un grupo de individuos con intervalos de referencia (IR) establecidos para animales de la región, e idealmente obtenidas con técnicas similares realizadas por el mismo laboratorio que generó los IR (Herdt y col 2000, Cozzi y col 2011). Amplios IR disminuyen la sensibilidad de los perfiles metabólicos, dificultando identificar rebaños con valores anormales y dando lugar a falsos negativos, mientras que IR estrechos reducen su especificidad, generando falsos positivos al clasificar rebaños normales como anormales (Horn y Pesce 2003, Van Saun 2010). Por otro lado, interpretar los resultados de un grupo de vacas utilizando IR generados para individuos no es apropiado. Esto se debe a que si el valor medio de la concentración de un mineral en un grupo de vacas coincide con el establecido en el límite inferior de referencia, la mitad de los animales tendrían concentraciones menores a lo adecuado si los datos presentan una distribución normal (Herdt y col 2000). Por lo tanto, la correcta interpretación de los perfiles metabólicos requiere del empleo de IR apropiados (Horn y Pesce 2003, Van Saun 2010), determinados específicamente para grupos de vacas lecheras. En relación con este aspecto, y con el objeto de minimizar la variación dentro del rebaño, es fundamental agrupar a los individuos en base a factores que puedan afectar las concentraciones de los parámetros, entre los que destaca su estado fisiológico (Herdt y col 2000, Van Saun 2010, Cozzi y col 2011). Sin embargo, actualmente, no se encuentran disponibles IR de minerales que se hayan establecido para grupos de vacas lecheras considerando estos factores. En este contexto, el objetivo del estudio fue proponer, para grupos de vacas preparto y en lactación de rebaños lecheros en el sur de Chile, IR de parámetros sanguíneos indicadores del balance mineral comúnmente evaluados en perfiles metabólicos.

\section{MATERIAL Y MÉTODOS}

Se utilizaron los valores de calcio $(\mathrm{Ca})$, fosfato inorgánico $(\mathrm{Pi})$, cociente calcio:fosfato inorgánico $(\mathrm{Ca}: \mathrm{Pi})$, magnesio $(\mathrm{Mg})$, sodio $(\mathrm{Na})$, potasio $(\mathrm{K})$, cobre $(\mathrm{Cu})$ y zinc (Zn) obtenidos de 1.414 perfiles metabólicos realizados a grupos de vacas en preparto $(n=380)$ y en lactación $(n=1.034)$ entre enero de 2003 y julio de 2011.

Los perfiles metabólicos fueron procesados en el Laboratorio de Patología Clínica Veterinaria, Facultad de Ciencias Veterinarias de la Universidad Austral de Chile (UACh), a partir de muestras sanguíneas remitidas para el control del balance metabólico nutricional y/o determinación de desbalances metabólicos en los rebaños lecheros. Se seleccionaron para el estudio aquellos perfiles que estaban constituidos por un grupo de 5 o más vacas, habitualmente 7, provenientes de rebaños lecheros ubicados en las regiones de La Araucanía, Los Ríos y Los Lagos (384' a $41^{\circ} 28^{\prime}$ LS y $72^{\circ} 35^{\prime}$ a $72^{\circ} 56^{\prime}$ LO), Chile, y que contaban con información sobre las características del grupo (preparto o lactación). Las muestras de elección, técnicas analíticas y equipos utilizados en el laboratorio para la determinación de los parámetros se indican en el cuadro 1. Si bien no se conocía en la mayoría de los perfiles analizados la raza, edad de los animales, las razas preponderantes en los sistemas productivos de las regiones consideradas en el estudio son la Holstein Frisian, Overo Colorado y Overo Negro. Aquellos perfiles en que

Cuadro 1. Muestras de elección, técnicas analíticas, coeficiente de variación (CV) de la técnica analítica y equipos utilizados para la determinación de las concentraciones sanguíneas de Ca, Pi, Mg, Na, K, Cu y Zn entre enero de 2003 y julio de 2011.

Sample, analytical techniques, coefficient of variation (CV) of the analytical technique and equipment used for the determination of blood concentrations of Ca, Pi, Mg, Na, K, Cu and Zn between January 2003 and July 2011.

\begin{tabular}{|c|c|c|c|c|c|}
\hline Analito & Técnica analítica & CV (\%) & Muestra elección & Período & Equipo \\
\hline $\mathrm{Ca}$ & EAA* $^{*}, 422,7 \mathrm{~nm}$ & 7,33 & \multirow{4}{*}{$\begin{array}{l}\text { Suero o plasma } \\
\text { heparinizado** }\end{array}$} & \multirow{6}{*}{$\begin{array}{l}01 / 2003-05 / 2007 \\
06 / 2007-07 / 2011\end{array}$} & \multirow{6}{*}{$\begin{array}{c}\text { EAA Perkin-Elmer } 2380 \\
\text { EAA Thermo Electron Corporation }{ }^{\circledR}, \mathrm{S} \text { Serie }\end{array}$} \\
\hline $\mathrm{Mg}$ & $\mathrm{EAA}^{*}, 285,2 \mathrm{~nm}$ & 8,31 & & & \\
\hline $\mathrm{Na}$ & $\begin{array}{l}\text { Fotometría de } \\
\text { llama, } 589 \mathrm{~nm}\end{array}$ & 5,37 & & & \\
\hline K & $\begin{array}{l}\text { Fotometría de } \\
\text { llama, } 766,5 \mathrm{~nm}\end{array}$ & 11,43 & & & \\
\hline $\mathrm{Zn}$ & $\mathrm{EAA}^{*}, 213,9 \mathrm{~nm}$ & 14,28 & Suero & & \\
\hline $\mathrm{Cu}$ & $\mathrm{EAA}^{*}, 324,5 \mathrm{~nm}$ & 13,11 & $\begin{array}{c}\text { Plasma } \\
\text { heparinizado** }\end{array}$ & & \\
\hline $\mathrm{Pi}$ & $\begin{array}{l}\text { Molibdato, } \\
\text { fotométrico, UV, } \\
340 \mathrm{~nm} \text {, art } 10027\end{array}$ & 6,81 & $\begin{array}{l}\text { Suero o plasma } \\
\text { heparinizado** }\end{array}$ & $\begin{array}{l}01 / 2003-05 / 2007 \\
06 / 2007-07 / 2011\end{array}$ & $\begin{array}{l}\text { Autoanalizador Roche }{ }^{\circledR}, \text { Cobas Mira Plus } \\
\text { Autoanalizador Wiener lab }{ }^{\circledast} \text {, Metrolab } 2300\end{array}$ \\
\hline
\end{tabular}

* EAA = espectrofotometría de absorción atómica.

** heparina de litio. 
los grupos de animales estaban constituidos por vaquillas fueron eliminados de los análisis.

Para controlar la precisión y exactitud de las técnicas empleadas en el análisis de las muestras se utilizó un suero control universal liofilizado, preparado de suero bovino con valores asignados para los componentes séricos (HumaTrol N, Human ${ }^{\circledR}$ ), y un pool plasmático o sérico preparado en el laboratorio a partir de muestras sanguíneas de vacas lecheras adultas. La precisión de las técnicas analíticas se evaluó con el coeficiente variación (CV), establecido a partir de valores obtenidos en el laboratorio entre los años 2008 y 2011 al controlar su exactitud mediante los sueros controles (cuadro 1).

El valor medio de la calcemia, fosfatemia, cociente $\mathrm{Ca}: \mathrm{Pi}$, magnesemia, natremia, kalemia, cupremia y zinquemia de cada grupo fue ingresado a una hoja Excel, Microsoft Office 2007, considerando la categoría del grupo (preparto o lactación).

\section{ANÁLISIS ESTADÍSTICO}

Los datos fueron analizados con el software MedCalc v.12.1.4 para Windows (MedCalc Software, Belgium). Los IR se establecieron para los grupos de vacas preparto y lactación en base a las normas de la American Society of Veterinary Clinical Pathology, ASVCP, (Friedrichs y col 2011) ${ }^{1}$. Inicialmente, se determinó la normalidad de los datos con la prueba de Kolmogorov-Smirnov, siendo los no normales normalizados por el método de logaritmos. Posteriormente, se evaluó la distribución de los datos y se identificaron los posibles outliers para cada parámetro mediante histogramas. Los outliers fueron analizados con el algortimo de Horn, basado en la determinación de los límites intercuartiles de Tukey y eliminados (Friedrichs y col 2011) $)^{1}$. Las pruebas estadísticas utilizadas para la obtención de los IR se seleccionaron en base al tamaño de la muestra. En aquellos casos en los cuales se disponía de un tamaño muestral $\geq 120$ los límites de referencia inferior (LIR) y superior (LSR) se calcularon con una prueba no paramétrica con base en los percentiles 2,5 y 97,5, respectivamente, no siendo necesaria la verificación del cumplimiento de los supuestos con respecto a la distribución de los datos; mientras que cuando el tamaño muestral fue $40 \leq n \leq 120$ se utilizó el método robusto para cálculo de valores referenciales según lo indicado por las normas de elaboración de valores referenciales, método que usa interacciones sucesivas hasta encontrar el centro y la dispersión de los datos pudiendo ser usado en ausencia de normalidad (Friedrichs y col 2011) ${ }^{1}$.

Friedrichs K, Harr KE, Freeman K, Szladovits B, Walton R, Barnhart K, Blanco J. 2011. ASVCP. Quality assurance and laboratory standards committee (QALS). Guidelines for the determination of reference intervals in veterinary species and other related topics. http://www.asvcp.org/pubs/pdf/RI\%20Guidelines\%20For\%20 ASVCP\%20website.pdf. 23 de enero de 2012.

\section{RESULTADOS Y DISCUSIÓN}

Las variables sanguíneas evaluadas correspondieron a los parámetros indicadores del balance mineral comúnmente analizados en los rebaños lecheros del sur de Chile. Los datos obtenidos a partir de los perfiles metabólicos realizados a grupos de vacas preparto y en lactación se presentan en el cuadro 2.

Los IR de la mayoría de los analitos fueron similares a los reportados y utilizados en el laboratorio para el análisis de los perfiles metabólicos (Wittwer 2004). Sin embargo, al comparar los IR de Pi para los grupos de vacas en preparto y en lactación y de Cu para los grupos en preparto con los IR descritos con anterioridad se observaron que los valores de $\mathrm{Pi}$ aumentaron y los de $\mathrm{Cu}$ disminuyeron. Esto podría deberse a cambios en sus concentraciones sanguíneas, asociadas al manejo nutricional de los rebaños y a que los IR fueron determinados para grupos de vacas y no individuos, diferenciándolos según su estado fisiológico; sin embargo no se cuenta con la información nutricional de las vacas. Es así como las mayores concentraciones de Pi establecidas como LIR y LSR en ambas categorías se asociarían a un incremento en la fosfatemia, que se refleja en la elevada presentación de hiperfosfatemia en los rebaños lecheros del sur de Chile en los últimos años (Barboza y col 2009, Céspedes 2011, Sepúlveda y col 2011), situación que podría afectar el cociente entre $\mathrm{Ca}$ y $\mathrm{Pi}$ en sangre. Sin embargo en la literatura no se cuenta con valores del cociente entre Ca y Pi que puedan ser comparados con el del presente estudio. En el caso del $\mathrm{Cu}$, el menor IR observado en la categoría preparto, en relación al IR descrito por Wittwer (2004) y el generado en este estudio para la categoría lactación, podría atribuirse a los mayores requerimientos de este microelemento descritos para vacas lecheras en avanzada gestación (NRC 2001). De este modo los resultados concuerdan con la menor cupremia reportada en vacas con $7-9$ meses de gestación, en comparación con vacas en lactación, en rebaños lecheros del sur de Chile (Wittwer y col 1988).

Es preciso tener en cuenta que los IR generados en este estudio se obtuvieron a partir de los registros de los perfiles metabólicos existentes en el laboratorio, lo cual puede ser un factor limitante. Por este motivo, la información acerca del estado sanitario y nutricional de los rebaños muestreados y la metodología empleada para la obtención y manejo de las muestras previo a su análisis era escasa o nula en muchas oportunidades. Por lo tanto, la identificación y eliminación de los outliers permitió excluir valores atípicos que podrían atribuirse al análisis de muestras de inadecuada calidad, contaminadas con microelementos, o provenientes de grupos de vacas con desbalances minerales, así como también valores asociados a errores de transcripción. Por otro lado, según las normas de la American Society of Veterinary Clinical Pathology (ASVCP) (Friedrichs y col 2011) ${ }^{1}$ y lo señalado por Horn y Pesce (2003), el tamaño muestral fue adecuado para estimar los IR de las variables. Sin embargo, los elevados CV de las técnicas 


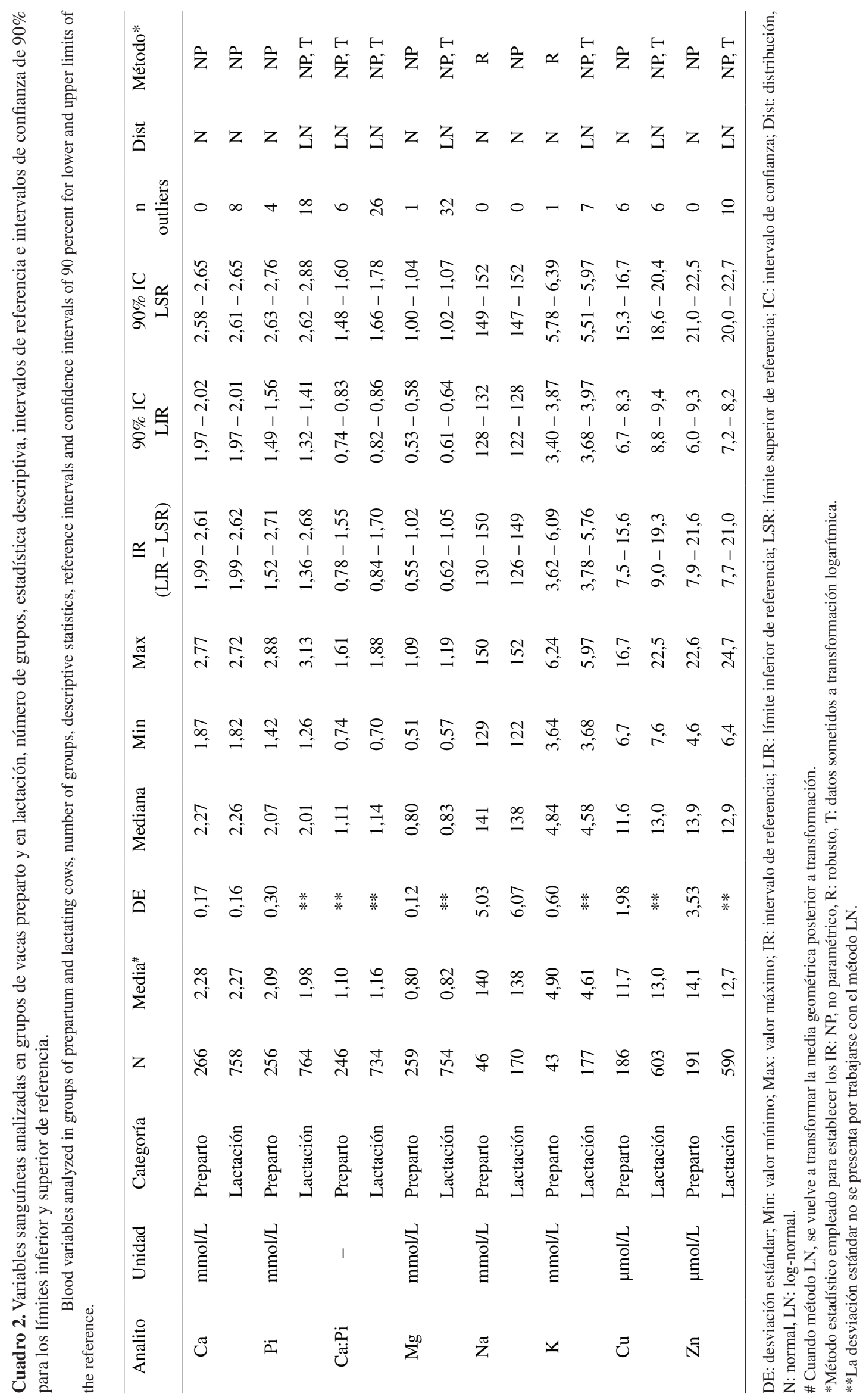


analíticas, observados principalmente en el caso del $\mathrm{K}, \mathrm{Cu}$ y $\mathrm{Zn}$, indican una baja precisión en la determinación de los analitos en las muestras sanguíneas, lo cual amplía los IR generados en el estudio (Friedrichs y col 2011) ${ }^{1}$.

Comparar los IR establecidos en este estudio con los obtenidos en el extranjero también presenta limitaciones, ya que existen variaciones en las concentraciones sanguíneas de los minerales asociadas al manejo nutricional de los rebaños, así como a condiciones edafoclimáticas (Wittwer y col 1987, Khan y col 2004). Además, el tipo de muestra y la técnica analítica utilizada en el laboratorio para la determinación de los analitos puede tener un efecto sobre los resultados obtenidos. Por otro lado, distintos métodos estadísticos pueden ser empleados para la determinación de los IR, sin embargo actualmente la comunidad científica indica el uso de la metodología aplicada en el presente estudio (Friedrichs y col 2011). Herdt y col (2000) sugieren que los IR de minerales para grupos de vacas lecheras dependen del número de animales a partir de los cuales se obtienen las muestras y de la desviación estándar de cada grupo.

Finalmente, se debe considerar que pocos laboratorios en la actualidad disponen de IR para grupos de vacas lecheras que se ajusten a los efectos del estado fisiológico de los animales (Van Saun 2010) y la literatura asociada es escasa. Por lo tanto, la utilización de los IR establecidos para grupos de vacas preparto y en lactación en este estudio contribuye a mejorar la interpretación y exactitud de los perfiles metabólicos para evaluar el estado metabólico nutricional de los rebaños lecheros en el sur de Chile.

\section{AGRADECIMIENTOS}

Se agradece la colaboración de la Tecnólogo Médico Helga Böhmwald y al Laboratorio de Patología Clínica Veterinaria, Facultad de Ciencias Veterinarias de la Universidad Austral de Chile, por haber facilitado la información de los perfiles metabólicos analizados en este estudio. Al Proyecto Consorcio Lechero FIA-CS-C-2004-1-P-M2P6, y a ANASAC, Chile por la beca de Magíster de C Wagemann.

\section{REFERENCIAS}

Barboza C, H Bohmwald, F Wittwer, PA Contreras, R Chihuailaf, M Noro. 2009. Razón Ca:Pi en sangre de vacas de rebaños lecheros en Chile: valores de perfiles metabólicos realizados entre 1986 - 2009. Resúmenes de XI Jornadas
Chilenas de Buiatría, Osorno, Chile, Pp 104-105.

Céspedes JA. 2011. Presentación de alteraciones metabólico-nutricionales en rebaños lecheros pastoriles de cinco macrozonas en el sur de Chile. Tesis de Magíster, Facultad de Ciencias Agrarias, Universidad Austral de Chile, Valdivia, Chile.

Cozzi G, L Ravarotto, F Gottardo, AL Stefani, B Contiero, L Moro, M Brscic, P Dalvit. 2011. Reference values for blood parameters in Holstein dairy cows: Effects of parity, stage of lactation, and season of production. J Dairy Sci 94, 3895-3901.

Herdt TH, W Rumbeiha, WE Braselton. 2000. The use of blood analyses to evaluate mineral status in livestock. Vet Clin North Am Food Anim Pract 16, 423-444.

Horn PS, AJ Pesce. 2003. Reference intervals: an update. Clin Chim Acta 334, 5-23.

Khan ZI, A Hussain, M Ashraf, MY Ashraf, M Yousaf, M Akhtar, A Maqbool. 2004. A review on mineral imbalances in grazing livestock and usefulness of soil, dietary components, animal tissues and fluid analysis in the assessment of these imbalances. J Anim Vet Adv 3, 394-412.

Laven RA, KE Lawrence, CT Livesey. 2007. The assessment of blood copper status in cattle: A comparison of measurements of caeruloplasmin and elemental copper in serum and plasma. $N$ Zeal Vet $J$ 55, 171-176.

NRC, National Research Council. 2001. Minerals. In: NRC (eds). Nutrient Requirements of Dairy Cattle. $7^{\text {th }}$ ed. National Academy Press, Washington DC, USA, Pp 105161.

Sepúlveda P, F Wittwer, H Böhmwald, M Noro. 2011. Relación entre la hiperfosfatemia y la hipocalcemia subclínica en vacas lecheras. Rev Arg Prod Anim 31, 39.

Van Saun RJ. 2010. Indicators of dairy cow transition risks: metabolic profiling revisited. In: Wittwer F, Chihuailaf R, Contreras H, Gallo C, Kruze J, Lanuza F, Letelier C, Monti G, Noro M (eds). Updates on Ruminant Production and Medicine. Andros Impresiones, Santiago, Chile, Pp 65-77.

Wittwer F, H Böhmwald, PA Contreras, J Filosa. 1987. Análisis de los resultados de perfiles metabólicos obtenidos en rebaños lecheros en Chile. Arch Med Vet 19, 35-45.

Wittwer F, P Contreras, H Böhmwald, R Anrique, R Fuchslocher. 1988. Concentraciones de zinc y cobre en forrajes $\mathrm{y}$ suero sanguíneo de 40 predios lecheros de la X Región Chile. Arch Med Vet 20, 118-125.

Wittwer F. 2004. Valores de referencia constituyentes bioquímicos sanguíneos en animales domésticos. Laboratorio de Patología Clínica Veterinaria, Universidad Austral de Chile, Valdivia, Chile.

Wittwer F. 2007. Diagnóstico y control de carencias minerales en bovinos. Seminario Internacional de Nutrición y Alimentación Animal UNISARC, Risaralda, Colombia. 
